\title{
Keyholes in MIMO-OFDM: Train-to-Wayside Communications in Railway Tunnels
}

\author{
Juan Moreno García-Loygorri, ${ }^{1}$ Leandro de Haro, ${ }^{2}$ José Manuel Riera, \\ Luis Cuéllar, ${ }^{2}$ and Carlos Rodríguez ${ }^{3}$ \\ ${ }^{1}$ Teoría de la Señal y Comunicaciones Department, ETSIS Telecomunicación, Universidad Politécnica de Madrid (UPM), \\ Campus Sur, Calle Nikola Tesla s/n, 28031 Madrid, Spain \\ ${ }^{2}$ ETSI Telecomunicación, Universidad Politécnica de Madrid (UPM), Ciudad Universitaria, 28040 Madrid, Spain \\ ${ }^{3}$ The Engineering Department of Metro de Madrid S.A., Madrid, Spain
}

Correspondence should be addressed to Juan Moreno García-Loygorri; juanmorenogl@diac.upm.es

Received 3 March 2017; Accepted 5 July 2017; Published 7 August 2017

Academic Editor: Yin Xuefeng

Copyright (C) 2017 Juan Moreno García-Loygorri et al. This is an open access article distributed under the Creative Commons Attribution License, which permits unrestricted use, distribution, and reproduction in any medium, provided the original work is properly cited.

\begin{abstract}
One of the key challenges in railway engineering is how to provide passengers an efficient, secure, and safe service. To achieve this, operators and stakeholders demand robust and high-capacity train-to-wayside broadband radio. Current radio technologies implement MIMO (Multiple-Input Multiple-Output) technology, whose use requires a good characterization of the propagation. In this paper, the results of an experimental campaign on MIMO propagation in subway tunnels are presented. The campaign makes use of an OFDM testbed at roughly $600 \mathrm{MHz}$, allowing the measurement of the $2 \times 2$ channel transfer matrix under different conditions regarding polarization, antenna separation, tunnel cross section, power allocation algorithm, and so forth. Particular attention is paid to the probability of appearance of keyholes, which imply a severe degradation of the MIMO performance even when transmitter and receiver antennas are uncorrelated. The measurements are carried out using a train that travels at the normal operative velocities. As a result of the measurements, it has been found that the use of vertical polarization at both terminals is advantageous and that, on average but for a narrow margin, $\lambda$-spaced antennas outperform $\lambda / 2$-spaced ones $(90 \%$ capacities of 7.00 and $6.76 \mathrm{bps} / \mathrm{Hz}$, resp.), although the latter show a lower probability of keyholes. However, keyhole probabilities are always below $2 \%$, so their influence on the performance of the whole system is limited.
\end{abstract}

\section{Introduction}

Communication between trains and wayside is essential today. Services like CCTV, CBTC (Communications-Based Train Control), ERTMS (European Rail Traffic Management System), and push-to-talk radio telephony are a few of the most popular services in this area. There are many examples of wireless technologies that could be used such as GSMR (Global System for Mobile-Railways) and TETRA (TransEuropean Trunked Radio) or others based on the IEEE $802.11 \mathrm{a} / \mathrm{b} / \mathrm{g}$ standard. However, the needs of both railway operators and passengers are far more demanding. This leads to a different scenario, where old technologies do not address the requirements [1], and new, state-of-the-art technologies may occupy their place. Some good examples are LTE-A,
WiMAX, or even IEEE 802.11ac. LTE (and LTE-A as well) has been flagged to be the next key technology in the field of railway communications [2], but many operators around the world have opted instead for IEEE 802.11-based solutions. This is a very actual topic in the railway field, as shown by the first deliverable of the EU-funded Roll2Rail Project [3].

All of these recent technologies have one thing in common: they all use MIMO technology to increase their effective bitrate [4]. This technique has shown its potential in many applications and has received a lot of attention since its very beginning $[5,6]$. However, there are some scenarios where MIMO does not fit and one of them is any scenario displaying "keyhole effect." Therefore, this keyhole effect has to be characterized. 
This effect causes a decrease in the capacity of the whole channel even when both transmitter and receiver are uncorrelated. The academic definition of a keyhole is a simultaneous low spatial correlation and a low-rank channelmatrix (ideally, 1) [7]. This effect was first postulated theoretically [7] and then measured on a controlled environment [8].

In previous papers, we have measured the keyhole effect on a railway tunnel and related its probability to the cross section of the tunnel [9] as well as to the polarization setup [10]. In this paper, we provide more results on the keyhole effect and we also map the influence of other parameters on the MIMO capacity.

In this short review of the bibliography, we will only mention a few of the most relevant references: a pioneer paper was centered on narrowband measurements [11] and another one focused on polarization effects in tunnels [12] and broadband measurements [13]; however, they lack the involvement of any train. Moreover, all these papers depict MIMO measurements in tunnels, with no trains running in real in-operation conditions and without any study on the effect of the separation between antenna elements.

This paper is organized in the following way: in Section 2, the measurements' setup and a description of the scenario are given; in Section 3, channel estimation procedure, capacity calculation, and keyhole determination are explained; in Section 4, results are presented and, finally, the main conclusions are summarized in Section 5.

\section{Measurement Setup and Description of the Scenario}

2.1. Setup: Testbed and Antenna Array. To perform these measurements, we used a MIMO testbed developed by some of the members of the team. It is described in full detail in [14], so in this section we will only highlight the key aspects of the setup. This testbed consists of a DVB-T2 [15] transmitter and receiver, in addition to two antennas on each side. The reason behind choosing DVB-T2 was that the transmission technology is OFDM (the same as LTE's downlink), and the frequency is similar to that of some bands $(700$ and $800 \mathrm{MHz})$ that will be used for LTE transmission. The frame structure of this testbed is based on DVB-T2, with some changes enabling implementing a $2 \times 2$ MIMO setup instead of a $2 \times 1$ MISO. The main parameters of the testbed are shown in Table 1.

User data is encoded using a distributed Alamouti [16] scheme. In this scheme, one branch transmits unmodified symbols, while the other only transmits modified symbols.

In this research work, we employed short-dipole arrays (the length of each antenna element is $22 \mathrm{~cm}$ ), matched at the desired frequency band $(594 \mathrm{MHz})$. Besides being close to the 700 and $800 \mathrm{MHz}$ bands, this frequency is not far from some interesting bands from a railway's point of view, like the GSM$\mathrm{R}$ band (876-880 and $921-925 \mathrm{MHz})$ or TETRA $(400 \mathrm{MHz}$ band).

To perform a complete measurement, it was required to place the transmitter onboard and the receiver in the platform and then the opposite: receiver onboard and transmitter in the platform. The onboard array was placed in the cabin's
TABLE 1: Main parameters of the MIMO testbed.

\begin{tabular}{lcc}
\hline Parameter & Symbol & Value \\
\hline FFT mode & - & $2 \mathrm{~K}$ \\
MIMO setup & - & $2 \times 2$ \\
Guard interval & $\mathrm{GI}$ & $1 / 8$ \\
Scattered pilot pattern & & $\mathrm{PP} 1$ \\
Frequency & $f_{c}$ & $594 \mathrm{MHz}$ \\
Modulation & - & $64 \mathrm{QAM}$ \\
Sampling frequency & $f_{s}$ & $9.1429 \mathrm{MHz}$ \\
Useful symbol time & $T_{u}$ & $2048 / F_{s}=224 \mu \mathrm{s}$ \\
Guard time & $T_{g}$ & $T_{s} / 8=28 \mu \mathrm{s}$ \\
Symbol time & $T_{s}$ & $252 \mu \mathrm{s}$ \\
Bandwidth & $\mathrm{BW}$ & $8 \mathrm{MHz}$ \\
Code & - & Distributed Alamouti \\
Data subcarriers & $N_{d}$ & 1878 \\
Carrier spacing & $\Delta f$ & $4.26 \mathrm{KHz}$ \\
\hline
\end{tabular}

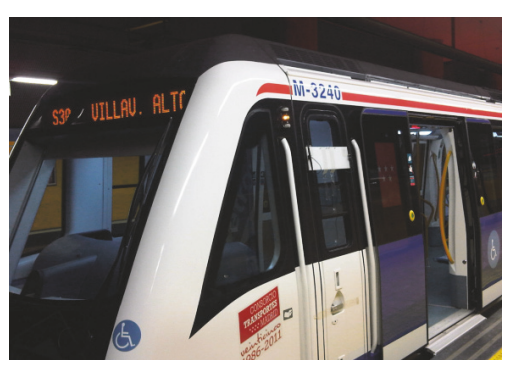

FIGURE 1: Rolling stock of the 3000-2 series.

window (see Figure 1) and the wayside array was located in the platform (see Figures 2 and 3). Thus, the onboard array was parallel to the axis of the tunnel in a very unfavorable way. The platform array forms a 30-degree angle with the axis of the tunnel. This setup (almost worst case) of the onboard system was intended to avoid permanent installations on the rolling stock and to test the system in as much real conditions as possible.

Measurements were carried out with two different spacings within antenna elements, $\lambda(51 \mathrm{~cm})$ and $\lambda / 2(25.5 \mathrm{~cm})$, and with copolarization (vertical-vertical and horizontalhorizontal) and cross-polarization (vertical-horizontal and horizontal-vertical) setups.

To properly address all the complexities related to tunnel propagation, we performed the measurements on an old man-made tunnel, with frequent changes on its section. Figure 4 shows these sections and the dimension of each tunnel stretch. This is a very typical scenario in many subway lines that were constructed prior to the invention of tunnel boring machines. In 571 meters, we can find up to six different tunnel shapes plus one slight curve (see Figure 5) and a gradient of 40 millimeter per meter in average.

\section{Data Model and Signal Processing}

3.1. Channel Estimation. It is a well-known fact that the performance of a MIMO system depends heavily on the 


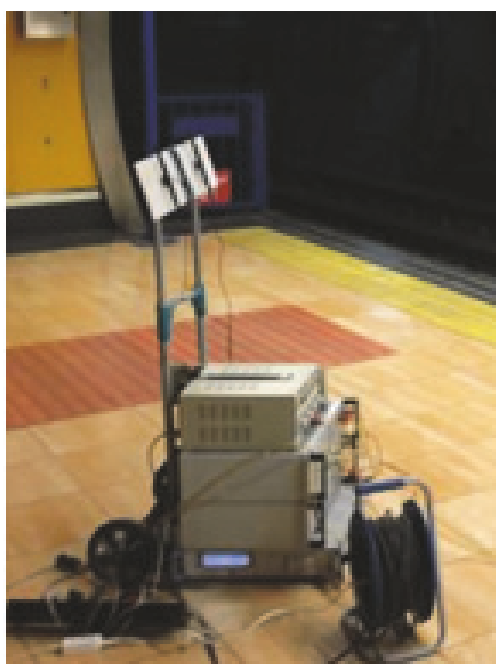

FIGURE 2: Receiver package.

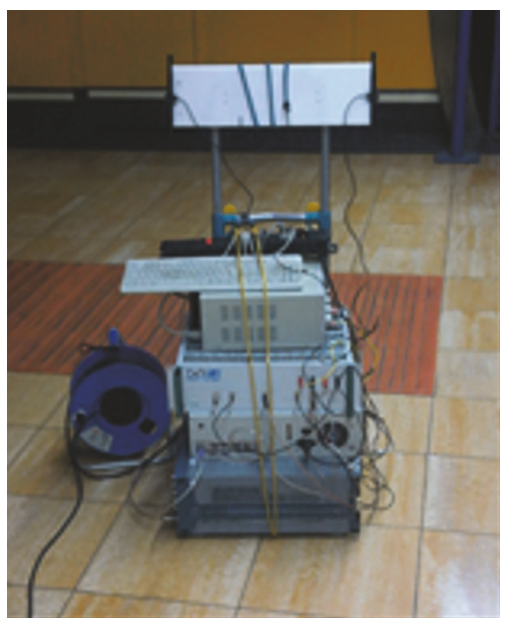

FIGURE 3: Transmitter package.

accuracy of the estimated channel matrix. Regarding the scattered pilot pattern, the DVB-T2 standard [15] proposes eight different patterns based on FFT size and GI. We decided to make use of the $2 \mathrm{~K}$ mode and a GI of $1 / 8$, so the scattered pilot pattern (for MISO) is the PP1 one. For each OFDM symbol, scattered pilots were placed every 12 subcarriers. In the time domain, the pilots begin in the first subcarrier and the initial point is shifted every 2 subcarriers for the following three OFDM symbols. So, the pilot structure consists of 4symbol blocks in time domain, being different in antenna 1 and antenna 2. In the case of antenna 2, the pilots for symbols 1 and 3 are inverted to figure out the transmitter antenna. Pilots are generated using a pseudorandom binary sequence (PRBS).

The data model is shown in (1), where $k$ is the subcarrier index (we have $N_{d}$ subcarriers); $X_{k}$ represents the transmitted data symbol; $N_{k}$ is the noise of subcarrier $k$; $H_{k}$ is the MIMO channel matrix and $Y_{k}$ is the received signal. So, on each symbol we transmit, we have $N_{d} H_{k}$ matrices. See Table 1 for the actual values of all these parameters.

$$
\begin{aligned}
Y_{k} & =H_{k} X_{K}+N_{k}, \\
H_{k} & =\left[\begin{array}{ll}
h_{1,1, k} & h_{1,2, k} \\
h_{2,1, k} & h_{2,2, k}
\end{array}\right] \\
& =\left[\begin{array}{ll}
\frac{r_{1, k}^{a}+r_{1, k}^{b}\left(x_{1, k}^{a} / x_{1, k}^{b}\right)}{2 x_{1, k}^{a}} & \frac{r_{1, k}^{a}-r_{1, k}^{b}\left(x_{1, k}^{a} / x_{1, k}^{b}\right)}{-2 x_{1, k}^{a}} \\
\frac{r_{2, k}^{a}+r_{2, k}^{b}\left(x_{1, k}^{a} / x_{1, k}^{b}\right)}{2 x_{1, k}^{a}} & \frac{r_{2, k}^{a}-r_{2, k}^{b}\left(x_{1, k}^{a} / x_{1, k}^{b}\right)}{-2 x_{1, k}^{a}}
\end{array}\right] .
\end{aligned}
$$

To estimate $H_{k}$ (the MIMO channel matrix), we use a scheme specifically developed for DVB-T2 [14], where $r_{1, k}$ and $r_{2, k}$ are the received symbols for antenna 1 and 2, respectively, for each subcarrier $k$. The superscripts " $a$ " and " $b$ " are related to cases A and B (in case B, both antenna 1 and antenna 2 have noninverted scattered pilots, and case A has one antenna with an inverted pilot and the other one noninverted). This procedure should be followed for each subcarrier of each received symbol while taking care of the coherence time of the channel (which has to be larger than $T_{s}$ ). In this case, $T_{s}$ is $252 \mu \mathrm{s}$ and the coherence time of the channel is around $1 \mathrm{~ms}$.

3.2. Capacity Calculation. Channel-matrix normalization is performed using Frobenius Norm at every realization of $H$ (each subcarrier of each symbol). Capacity was computed assuming equal-power allocation, so for MIMO channels it follows expression (3) taken from [5]. Therefore, for every $k$, the capacity $C_{k}$ is

$$
C_{k}=\log _{2}\left[\operatorname{det}\left(I_{M}+\frac{\mathrm{SNR}}{N} H_{k} H_{k}^{H}\right)\right] .
$$

$M$ and $N$ are the receiving and transmitting antenna elements, respectively, and $H^{H}$ is the transpose conjugate (Hermitian) of $H$. $I_{M}$ is the $M \times M$ identity matrix (in this case, $M=N=2$ ).

3.3. Keyhole Determination. The physical definition of a keyhole consists in having low spatial correlation and, in an apparent paradox, also a channel matrix with a low rank (ideally 1 , which implies no diversity). At first it was postulated [7] and then measured on a controlled environment [8] (a narrow hole in a wall between transmitter and receiver). But noise complicates heavily this theoretical scenario due to the lack of matrix ranks equal to one. Therefore, we shall estimate a keyhole with a different procedure:

$$
\frac{\max \left(\lambda_{1}, \lambda_{2}\right)}{\min \left(\lambda_{1}, \lambda_{2}\right)} \geq \eta \longrightarrow \text { keyhole. }
$$

$\lambda_{1}$ and $\lambda_{2}$ are the two eigenvalues of the $2 \times 2$ correlation matrix $H H^{H} . \eta$ is an arbitrary threshold that sometimes takes the same value as the SNR (this was the approach the first time a keyhole was measured [8]). In our case, we 


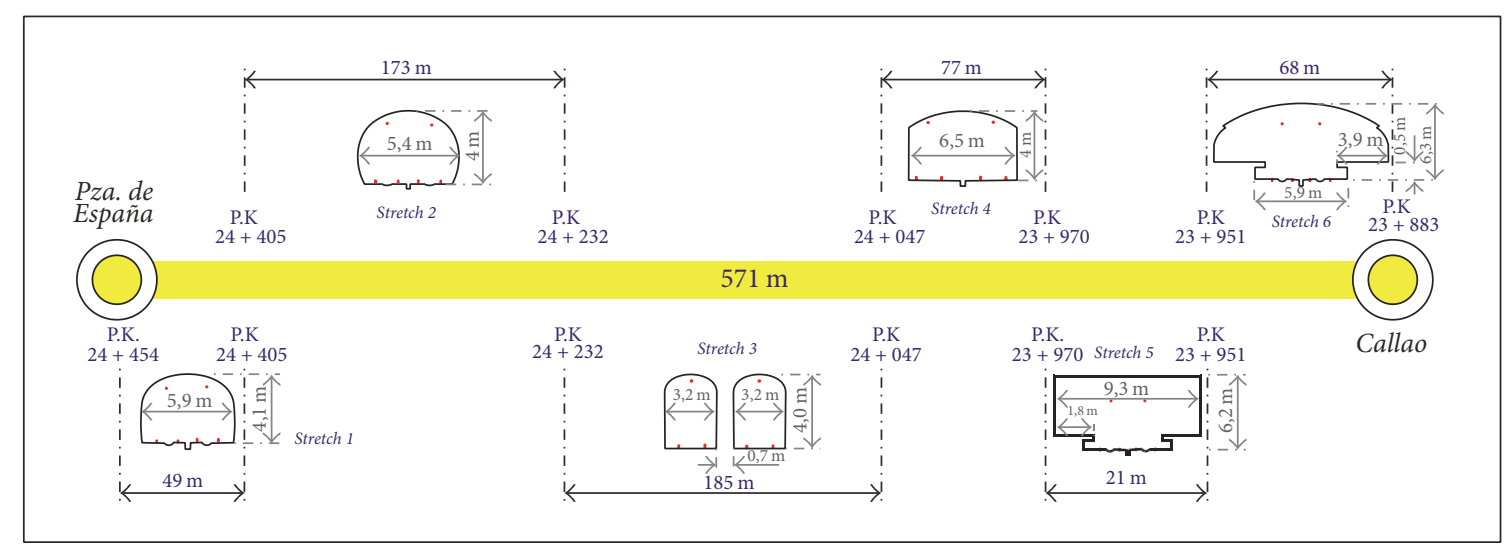

FIgUre 4: Tunnel cross sections (Callao-Plaza de España).

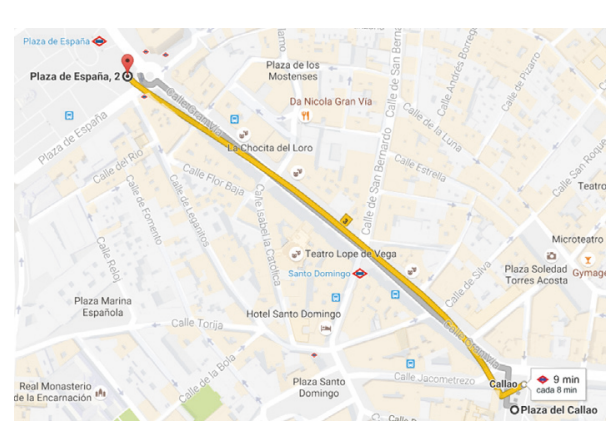

FIGURE 5: Floor plan of the track Callao-Plaza de España, showing a slight curve between them.

decided to have $\eta=20 \mathrm{~dB}$ as this is the largest SNR measured in the entire track (excluding the LOS measurements not considered here). Therefore, our approach is the same as in [8] but following a more conservative conception of the threshold (that leads to a higher value for $\eta$ ) in order to not overestimate the amount of keyholes.

Keyhole probability is presented here as a ratio between the measured keyholes and the total number of realizations of $H$ which are measured. It is expected to degrade the average capacity of the $2 \times 2$ MIMO system, because every time we have a keyhole, the $2 \times 2$ MIMO system has a performance as a $1 \times 1$ SISO one.

Also, when condition (4) is fulfilled, we need to check out that the local correlation is low (below 0.75 [9]). Otherwise, we would not have a keyhole but a largely correlated array instead. With this approach, we separate the effect of the lowrank criterion in (4) and the correlation between the antenna elements in the keyhole probability.

\section{Experimental Results}

4.1. Measurements. We carried out a measurement campaign in Line 3 of Madrid Metro between the stations of Callao and Plaza de España. In Figure 4, the cross sections of the tunnels are shown. Unless stated otherwise, every result is the average of two different measurements: one for the
TABLE 2: $C_{90}(\mathrm{bps} / \mathrm{Hz})$ for $\mathrm{VV}, \mathrm{VH}, \mathrm{HV}$, and $\mathrm{HH}$ polarization setups for both antenna separations considered $(\lambda$ and $\lambda / 2)$.

\begin{tabular}{lccccc}
\hline $\begin{array}{l}\text { Antenna } \\
\text { distance/polarization }\end{array}$ & $\mathrm{VV}$ & $\mathrm{HV}$ & $\mathrm{VH}$ & $\mathrm{HH}$ & Average \\
\hline$\lambda$ & 7.23 & 7.15 & 6.82 & 6.78 & 7.00 \\
$\lambda / 2$ & 6.79 & 6.91 & 6.68 & 6.66 & 6.76 \\
\hline
\end{tabular}

uplink and the other for the downlink. Furthermore, every measurement was under NLOS conditions, with the train running under real in-operation conditions. These real inoperation conditions were guaranteed by the ATO (Automatic Train Operation) subsystem, which is part of the signaling system and controls the speed of the train. ATO system also guaranteed that every measurement was carried out at the same speed $( \pm 2 \mathrm{~km} / \mathrm{h})$. The train employed in the measurement campaign belonged to the 3000 series by CAF. Its highest speed at commercial service is $80 \mathrm{~km} / \mathrm{h}$. The train car body shell is made of aluminum.

4.2. Results. The measured results are capacity in $\mathrm{bps} / \mathrm{Hz}$ as described in (3) and keyhole probability (4). We will show the impact of polarization on capacity and the effects of the antenna separation on both capacity and keyhole probability. We will consider two antenna-element separations ( $\lambda$ and $\lambda / 2$ ) and four polarization setups (VV, VH, HV, and $\mathrm{HH}$ ).

4.2.1. Capacity. To get an idea of the average performance of the system, a suitable parameter is the capacity exceeded in $90 \%$ of the measurements $\left(C_{90}\right)$, which is shown in Table 2 for all the measurements included in this paper. In Figure 6, an example of one of these measurements is depicted (as a CDF plot). Figure 7 shows $6 \mathrm{CDF}$ plots of the measured capacity: two $1 \times 1$ SISO (VV and $\mathrm{HH}$ ) and four $2 \times 2$ MIMO curves ( $\mathrm{HV}, \mathrm{VV}, \mathrm{HH}$, and $\mathrm{VH}$ ).

On average but for a narrow margin, $\lambda$-spaced antennas outperform $\lambda / 2$-spaced ones (7.00 and $6.76 \mathrm{bps} / \mathrm{Hz}$, resp.). This $3.4 \%$ difference is an expectable result, because it is a well-known fact that capacity increases as distance between antenna elements becomes larger. 


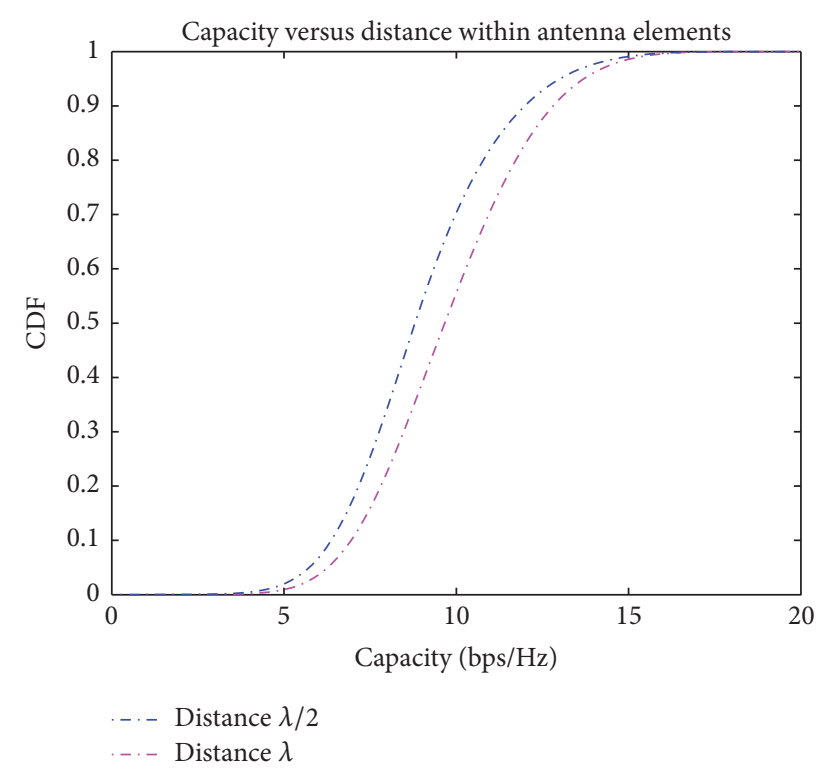

FIGURE 6: CDF capacity versus antenna separation within the array (bps/Hz).

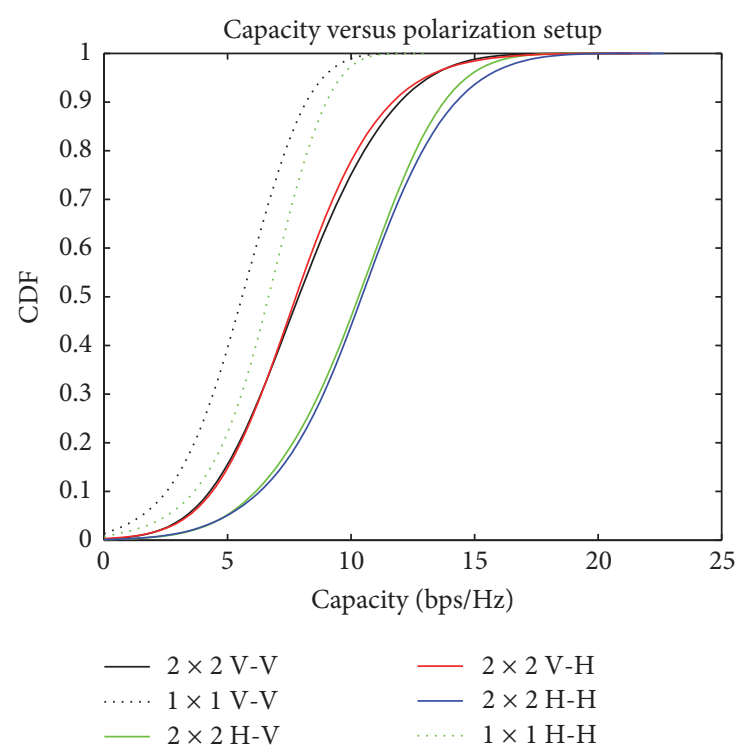

FIgURE 7: CDF capacity versus polarization setup for $\lambda$-spaced antenna elements $(\mathrm{bps} / \mathrm{Hz})$.

The best setup in terms of capacity is VV polarization with $\lambda$-spaced antennas, closely followed by $\mathrm{HV} \lambda$-spaced antennas (7.23 and $7.15 \mathrm{bps} / \mathrm{Hz}$, resp.). The best setup of $\lambda / 2$-spaced antennas is $\mathrm{HV}$ and then $\mathrm{VV}$. We can conclude that when the receiver is vertically polarized ( $\mathrm{VV}$ and $\mathrm{HV}$ ), capacities are larger, because horizontally polarized receivers suffer higher attenuations for the presence of curves in tunnels [17]. This phenomenon happens in both $\lambda$ and $\lambda / 2$ spaced setups and is coherent with other measurements in the field [17] but only in terms of attenuation. Here we present results of keyhole probability and channel capacity. It is important to note that curve here means both in the horizontal plane (a "normal" curve) and also in the vertical
TABLE 3: Keyhole probabilities (\%) for VV, VH, HV, and $\mathrm{HH}$ polarization setups for both antenna separations considered $(\lambda$ and $\lambda / 2)$.

\begin{tabular}{lccccc}
\hline $\begin{array}{l}\text { Antenna } \\
\text { distance/polarization }\end{array}$ & VV & HV & VH & HH & Average \\
\hline$\lambda$ & $0.58 \%$ & $1.20 \%$ & $0.85 \%$ & $0.58 \%$ & $0.80 \%$ \\
$\lambda / 2$ & $0.34 \%$ & $0.94 \%$ & $0.66 \%$ & $0.48 \%$ & $0.60 \%$ \\
\hline
\end{tabular}

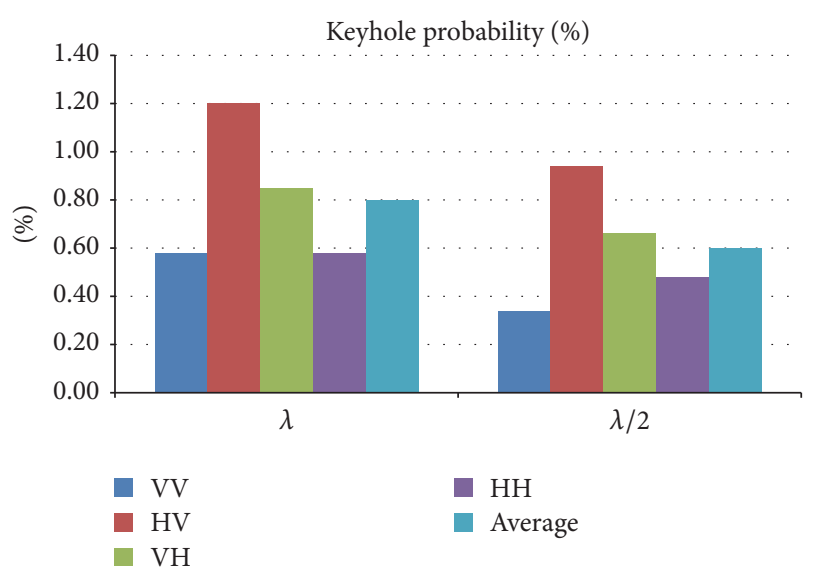

FIGURE 8: Keyhole probabilities versus antenna separation versus polarization setup.

plane (i.e., a gradient). In the track, we have a slight curve in the horizontal plane and an average gradient of $40 \mathrm{~mm} / \mathrm{meter}$. Each one of them impacts one polarization setup.

4.2.2. Keyhole Probability. The average keyhole probability for each type of tunnel considered was measured in our previous paper [9], where we found that the sections that were more likely to have keyholes were stretches 2 and 3 (see Figure 2 of [9]).

In Table 3 and Figure 8, average keyhole probabilities are shown for each setup. We can see that a cross-polarization setup, $\mathrm{HV}$ in $\lambda$-separated antennas, concentrates almost twice keyholes compared to the average of all the other setups of this separation. If we decrease our separation to $\lambda / 2$, what we observe is that the same setup (HV) outnumbers all the others but in a lesser extent. This result is coherent with our previous paper [10], where we measured that the polarization setup that concentrates more keyholes is HV. Copolarization setups ( VV and $\mathrm{HH}$ ) have less keyholes than their crosspolarization counterparts ( $\mathrm{VH}$ and $\mathrm{HV}$ ) and are very similar to each other when the antenna-element separation is the same (VV and $\mathrm{HH}, 0.58 \%$ in $\lambda ; 0.34 \%$ and $0.48 \%$, for $\mathrm{VV}$ and $\mathrm{HH}$, resp., in $\lambda / 2$ ). So, the relative performance (in terms of keyhole probability) of $\lambda$-separated arrays is the same as $\lambda / 2$ separated ones, but the latter have smaller probabilities.

In average, $\lambda$-spaced antennas suffer more keyholes than $\lambda / 2$-spaced ones $(0.80 \%$ and $0.60 \%$, resp.). This is a relative increase of $33.3 \%$. In every polarization setup, $\lambda$ spaced antennas have more keyholes than their $\lambda / 2$-spaced counterparts. This result is influenced by the fact that $\lambda$-spaced antennas have a higher local correlation, so it is 
more likely that when condition (3) is fulfilled, we are not in a keyhole scenario due to high correlation (i.e., we would have a low rank caused by high correlation instead of a keyhole).

Moreover, the largest differences between $\lambda$-spaced and $\lambda / 2$-spaced arrays exist in VV and VH setups, both in terms of capacity and keyhole probability. The reason behind this behavior is the presence of curves in the tunnel which downgrade the overall performance of horizontally polarized signals [17]. As explained before, the gradient is also a curve in the vertical plane, so vertically polarized signals suffer, too (but less than horizontal ones, because this curve has a smaller radius).

Another aspect that should be considered is that the impact of these keyholes on the performance of the whole system is low. Keyhole probabilities below $2 \%$ cannot lead to a significant decrease of the capacity, so their impact is negligible. This is the reason why (in average) a MIMO system with $\lambda$-spaced antennas has more keyholes and also larger capacities than the correspondent system but $\lambda / 2$-spaced antennas. Large keyhole probabilities and large capacities (at least a priori) are incompatible with each other but not if we take into account the fact that the absolute keyhole probabilities are very low and so not very influential in the whole performance.

Numerical results point out that the polarization at the transmitter is more influential on the keyhole probability and, in the same way, polarization at the receiver for the capacity.

\section{Conclusion}

The performance of a MIMO-OFDM train-to-wayside system has been appraised. Moreover, the likelihood of a physical phenomenon that affects the performance of a MIMO system (i.e., keyhole effect) has also been measured. These measurements were carried out at $594 \mathrm{MHz}$, using a $2 \times 2$ MIMO testbed on a train in real in-operation situation inside the tunnels. On the other hand, the research described in the paper can be related to the use of OFDM systems, such as LTE, in the context of railway applications using frequencies below $1 \mathrm{GHz}$.

We have related the influence of antenna-element separation to two key parameters: channel capacity and keyhole probability. The obtained results are coherent with our previous papers on this topic and give a deeper insight into the behavior of MIMO systems in tunnels. Moreover, we see that $\lambda$-spaced antennas provide larger capacities, whereas they suffer more keyholes than their $\lambda / 2$-spaced counterparts. This result is not contradictory when we take into account the fact that the observed keyhole probabilities are always below $2 \%$, so their influence on the performance of the whole system is limited. Therefore, the recommendation for train-to-wayside systems is to separate antenna elements as much as possible and also to work only in vertical polarization.

\section{Conflicts of Interest}

The authors declare that there are no conflicts of interest regarding the publication of this paper.

\section{Acknowledgments}

This work was supported in part by the Ministry of Economy and Competitiveness of Spain through Project TEC201457821-R. Leandro de Haro passed away on 30 July 2015. The three remaining coauthors of this article wish to express their gratitude to Leandro for his wonderful contributions and his research work in antennas and communications and also for being a fantastic person.

\section{References}

[1] J. Calle-Sanchez, M. Molina-Garcia, and J. I. Alonso, "Top challenges of LTE to become the next generation railway communication system," in Proceedings of the COMPRAIL 2012. 13th International Conference on Design and Operation in Railway Engineering, pp. 85-96, Wessex Institue of Technology, New Forest, UK, September 2012.

[2] D. Mandoc, "LTE/SAE-The Future Railway Mobile Radio System: Long-Term Vision on Railway Mobile Radio Technologies," Technical Report. UIC, 2009.

[3] D2.3 "State of the Art in Radio Technologies and Recommendation of Suitable Technologies", Roll2Rail Project - WP.2 TCMS, [online]: http://www.roll2rail.eu/download.aspx?id=26132f73a688-4dcc-bc77-56f9aab4ef78.

[4] Q. H. Li, G. J. Li, W. Lee et al., "MIMO techniques in WiMAX and LTE: a feature overview," IEEE Communications Magazine, vol. 48, no. 5, pp. 86-92, 2010.

[5] A. Goldsmith, S. A. Jafar, N. Jindal, and S. Vishwanath, "Capacity limits of MIMO channels," IEEE Journal on Selected Areas in Communications, vol. 21, no. 5, pp. 684-702, 2003.

[6] D. Gesbert, M. Shafi, D. S. Shiu, P. J. Smith, and A. Naguib, "From theory to practice: an overview of MIMO space-time coded wireless systems," IEEE Journal on Selected Areas in Communications, vol. 21, no. 3, pp. 281-302, 2003.

[7] D. Chizhik, G. J. Foschini, and M. J. Gans, "Keyholes, correlations, and capacities of multielement transmit and receive antennas," IEEE Transactions on Wireless Communications, vol. 1, no. 2, pp. 361-368, 2002.

[8] P. Almers, F. Tufvesson, and A. F. Molisch, "Keyhole effect in MIMO wireless channels: measurements and theory," IEEE Transactions on Wireless Communications, vol. 5, no. 12, pp. 3596-3604, 2006.

[9] J. Moreno, L. De Haro, C. Rodriguez, L. Cuellar, and J. M. Riera, "Keyhole estimation of an MIMO-OFDM train-to-wayside communication system on subway tunnels," IEEE Antennas and Wireless Propagation Letters, vol. 14, pp. 88-91, 2015.

[10] J. Moreno García-Loygorri, L. De Haro, C. Rodríguez, L. Cuéllar, and J. M. Riera, "Influence of polarization on keyhole probability on a MIMO-OFDM train-to-wayside system on tunnels," IEEE Antennas and Wireless Propagation Letters, vol. 14, pp. 1798-1801, 2015.

[11] M. Liénard, P. Degauque, J. Baudet, and D. Degardin, "Investigation on MIMO channels in subway tunnels," IEEE Journal on Selected Areas in Communications, vol. 21, no. 3, pp. 332-339, 2003.

[12] J.-M. Molina-García-Pardo, M. Lienard, P. Degauque, C. García-Pardo, and L. Juan-Llácer, "MIMO Channel capacity with polarization diversity in arched tunnels," IEEE Antennas and Wireless Propagation Letters, vol. 8, pp. 1186-1189, 2009. 
[13] C. Sanchis-Borras, J.-M. Molina-Garcia-Pardo, M. Lienard, and P. Degauque, "Performance evaluation of MIMO-OFDM in tunnels," IEEE Antennas and Wireless Propagation Letters, vol. 11, pp. 301-304, 2012.

[14] C. Gómez-Calero, L. C. Navarrete, L. De Haro, and R. Martínez, "A $2 \times 2$ MIMO DVB-T2 System: Design, new channel estimation scheme and measurements with polarization diversity," IEEE Transactions on Broadcasting, vol. 56, no. 2, pp. 184-192, 2010.

[15] Digital Video Broadcasting (DVB); Frame Structure Channel Coding and Modulation for a Second Generation Digital Terrestrial Television Broadcasting System (DVB-T2)," ETSI, EN 302755 V1.1.1 (2008-04).

[16] S. M. Alamouti, "A simple transmit diversity technique for wireless communications," IEEE Journal on Selected Areas in Communications, vol. 16, no. 8, pp. 1451-1458, 1998.

[17] J. A. Castiblanco, D. Seetharamdoo, M. Berbineau, M. Ney, and F. Gallee, "Determination of antenna specification and positioning for efficient railway communication in tunnels of arbitrary cross section," in Proceedings of the 11th International Conference on ITS Telecommunications (ITST '11), pp. 678-683, IEEE, St. Petersburg, Russia, August 2011. 


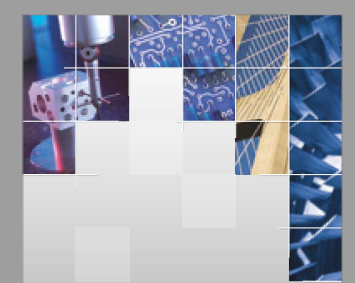

\section{Enfincering}
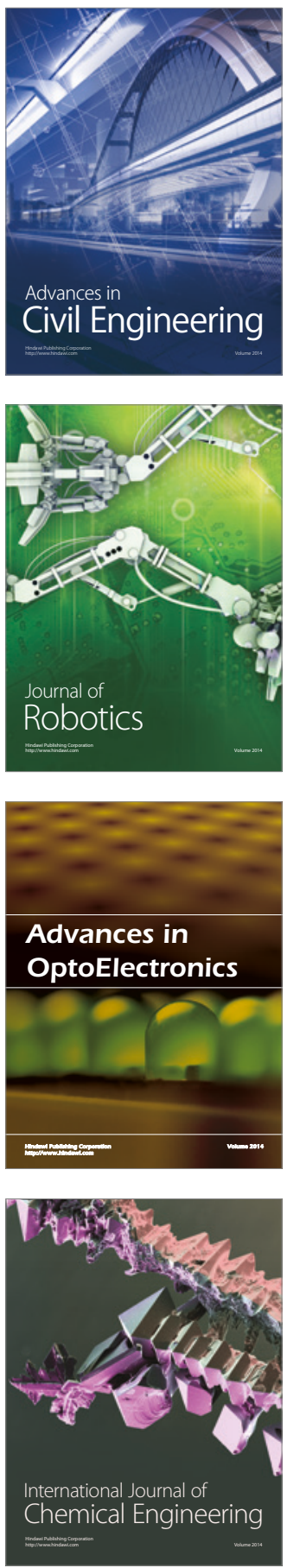

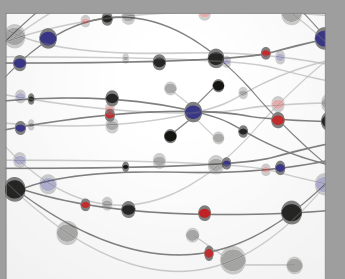

The Scientific World Journal

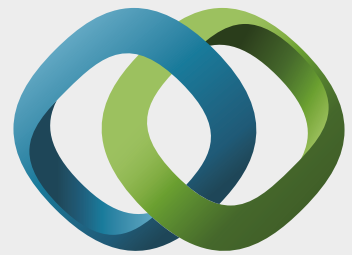

\section{Hindawi}

Submit your manuscripts at

https://www.hindawi.com
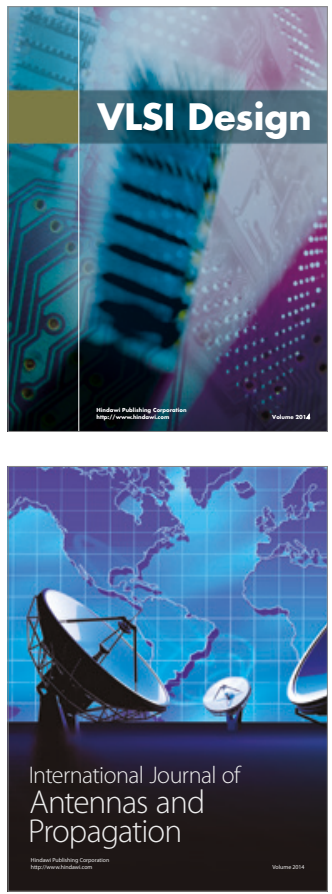

\section{Rotating}

Machinery
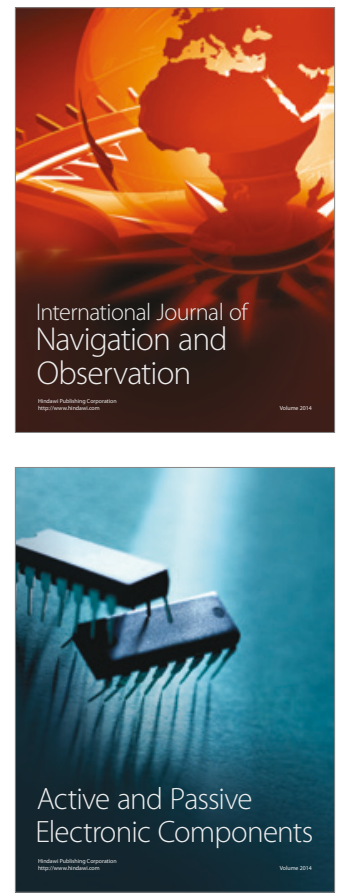
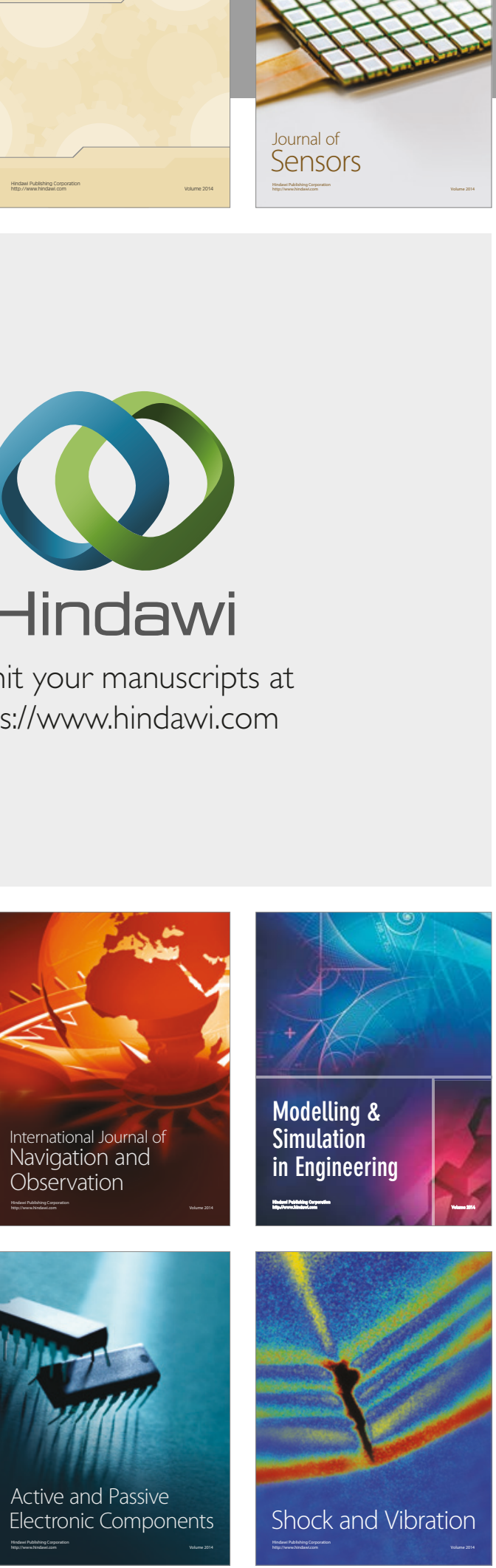
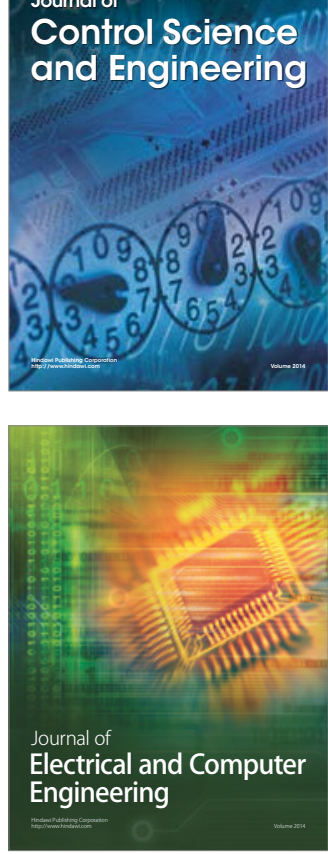

Distributed

Journal of

Control Science

and Engineering
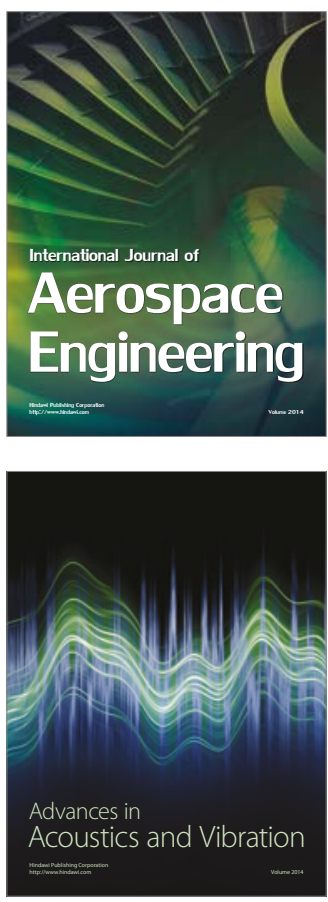

Sensor Networks 